Acta Crystallographica Section C

Crystal Structure

Communications

ISSN 0108-2701

Editor: George Ferguson

\title{
Diaquabis(2-quinolinecarboxylato- $\mathrm{N}, \mathrm{O}$ )manganese(II)
}

N. Okabe and M. Koizumi

This electronic document was scanned from an archival copy of material deposited to accompany a paper published in an IUCr journal. In many cases the only accessible copy was a microfilm of a poor-quality original. 


$$
83693
$$




\begin{tabular}{|c|c|c|c|c|c|c|c|c|c|c|c|c|c|c|}
\hline $\mathrm{k}$ & Fo & FC & $\operatorname{sigF}$ & $\mathrm{k}$ & 1 & FO & FC & sigt & $\mathrm{k}$ & 1 & FO & & $\mathrm{FC}$ & sig $F$ \\
\hline-7 & 75 & 66 & 3 & 7 & -4 & 109 & 108 & 3 & 11 & -5 & 161 & & 160 & 4 \\
\hline-6 & 118 & 113 & 3 & 7 & -3 & 125 & 124 & 3 & 11 & -4 & 43 & & 46 & 3 \\
\hline-5 & 103 & 99 & 3 & 7 & -1 & 218 & 214 & 4 & 11 & -3 & 183 & & 187 & 4 \\
\hline-4 & 568 & 560 & 9 & 7 & 0 & 1.68 & 166 & 3 & 11 & -1 & 235 & & 241 & 4 \\
\hline-3 & 225 & 220 & 4 & 7 & 1 & 385 & 379 & 6 & 11 & 1 & 198 & & 191 & 4 \\
\hline-2 & 254 & 265 & 4 & 7 & 2 & 195 & 185 & 3 & 11 & 2 & 114 & & 113 & 3 \\
\hline-1 & 1015 & 1025 & 15 & 7 & 3 & 37 & 25 & 3 & 11 & 3 & 116 & & 108 & 3 \\
\hline 0 & 416 & 400 & 6 & 7 & 4 & 64 & 66 & 3 & 11 & 4 & 74 & & 76 & 3 \\
\hline 1 & 323 & 305 & 5 & 7 & 5 & 160 & 155 & 4 & 11 & 5 & 152 & & 153 & 4 \\
\hline 2 & 186 & 182 & 3 & 7 & 6 & 37 & 43 & 4 & 11 & 6 & 43 & & 45 & 4 \\
\hline 3 & 50 & 53 & 3 & 7 & 7 & 90 & 93 & 3 & 12 & -6 & 106 & & 104 & 4 \\
\hline 4 & 289 & 285 & 5 & 8 & -8 & 117 & 112 & 4 & 12 & -5 & 53 & & 52 & 3 \\
\hline 5 & 28 & 28 & 5 & 8 & -6 & 144 & 143 & 4 & 12 & -4 & 41 & & 37 & 4 \\
\hline 6 & 182 & 181 & 4 & 8 & -4 & 113 & 110 & 3 & 12 & -3 & 67 & & 68 & 3 \\
\hline 7 & 46 & 39 & 4 & 8 & -3 & 33 & 36 & 4 & 12 & -2 & 248 & & 257 & 4 \\
\hline 8 & 36 & 28 & 5 & 8 & -2 & 180 & 188 & 3 & 12 & -1 & 123 & & 122 & 3 \\
\hline-8 & 58 & 62 & 3 & 8 & -1 & 434 & 440 & 7 & 12 & 0 & 98 & & 106 & 4 \\
\hline-7 & 73 & 74 & 3 & 8 & 0 & 238 & 252 & 4 & 12 & 1 & 38 & & 31 & 4 \\
\hline-6 & 162 & 157 & 4 & 8 & 1 & 79 & 73 & 3 & 12 & 2 & 206 & & 200 & 4 \\
\hline-5 & 273 & 274 & 5 & 8 & 2 & 203 & 207 & 4 & 12 & 3 & 49 & & 46 & 3 \\
\hline-4 & 129 & 133 & 3 & 8 & 3 & 98 & 95 & 3 & 12 & 4 & 128 & & 120 & 4 \\
\hline-3 & 311 & 314 & 5 & 8 & 4 & 105 & 102 & 3 & 12 & 6 & 34 & & 30 & 5 \\
\hline-2 & 324 & 316 & 5 & 8 & 5 & 42 & 43 & 3 & 13 & -5 & 79 & & 79 & 3 \\
\hline-1 & 507 & 523 & 8 & 8 & 6 & 139 & 140 & 4 & 1.3 & -4 & 90 & & 98 & 4 \\
\hline 1 & 300 & 301 & 5 & 8 & 7 & 33 & 28 & 5 & 13 & -3 & 78 & & 77 & 4 \\
\hline 5 & 85 & 82 & 3 & 9 & -7 & 110 & 103 & 4 & 13 & -1 & 103 & & 1.08 & 4 \\
\hline 5 & 156 & 163 & 3 & 9 & -6 & 51 & 57 & 3 & 13 & 0 & 86 & & 90 & 4 \\
\hline 5 & 53 & 54 & 3 & 9 & -5 & 155 & 156 & 4 & 13 & 1 & 142 & & 134 & 4 \\
\hline 5 & 168 & 166 & 3 & 9 & -4 & 127 & 131 & 3 & 13 & 3 & 94 & & 92 & 4 \\
\hline 5 & 188 & 184 & 4 & 9 & -3 & 344 & 360 & 6 & 13 & 5 & 36 & & 36 & 5 \\
\hline 5 & 171 & 170 & 4 & 9 & -2 & 150 & 154 & 3 & 14 & -5 & 33 & & 26 & 5 \\
\hline-8 & 75 & 69 & 3 & 9 & -1 & 157 & 171 & 3 & 14 & -3 & 116 & & 117 & 4 \\
\hline-7 & 68 & 65 & 3 & 9 & $\overline{0}$ & 165 & 177 & 3 & 14 & -2 & 126 & & 130 & 4 \\
\hline-6 & 210 & 207 & 4 & 9 & 1 & 303 & 304 & 5 & 14 & 0 & 54 & & 61 & 3 \\
\hline-5 & 56 & 61 & 3 & 9 & 3 & 151 & 148 & 3 & 14 & 1 & 56 & & 54 & 3 \\
\hline-4 & 279 & 288 & 5 & 9 & 5 & 230 & 229 & 4 & 14 & 2 & 150 & & 148 & 4 \\
\hline-3 & 96 & 100 & 3 & 9 & 6 & 54 & 51 & 3 & 14 & 4 & 103 & & 101 & 4 \\
\hline-2 & 484 & 501 & 7 & 9 & 7 & 66 & 59 & 3 & 15 & -1 & 121 & & 121 & 4 \\
\hline-1 & 43 & 43 & 2 & 10 & -7 & 37 & 25 & 5 & 15 & 0 & 57 & & 56 & 3 \\
\hline 0 & 373 & 380 & 6 & 10 & -6 & 92 & 94 & 4 & 15 & 1 & 106 & & 89 & 4 \\
\hline 6 & 45 & 46 & 2 & 10 & -4 & 103 & 109 & 4 & 15 & 3 & 136 & & 134 & 4 \\
\hline 6 & 241 & 238 & 4 & 10 & -3 & 78 & 78 & 3 & 15 & 4 & 36 & & 38 & 5 \\
\hline 6 & 260 & 240 & 4 & 10 & -2 & 144 & 144 & 3 & 16 & -2 & 39 & & 37 & 5 \\
\hline 6 & 224 & 217 & 4 & 10 & -1 & 103 & 100 & 3 & 16 & -1 & 37 & & 39 & 5 \\
\hline 6 & 270 & 270 & 5 & 10 & 0 & 133 & 136 & 3 & 16 & 0 & 60 & & 60 & 3 \\
\hline 6 & 45 & 34 & 4 & 10 & 1 & 43 & 37 & 3 & 16 & 1 & 49 & & 47 & 4 \\
\hline 6 & 99 & 86 & 4 & 10 & 2 & 197 & 190 & 4 & 16 & 2 & 70 & & 64 & 3 \\
\hline-7 & 107 & 101 & 4 & 10 & 3 & 121 & 111 & 3 & & & & & & \\
\hline-6 & 30 & 31 & 5 & 10 & 4 & 110 & 102 & 4 & & $\leadsto \mathrm{h}$ & $=$ & 2 & & \\
\hline-5 & 93 & 91 & 4 & 10 & 7 & 47 & 40 & 4 & & & & & & \\
\hline
\end{tabular}




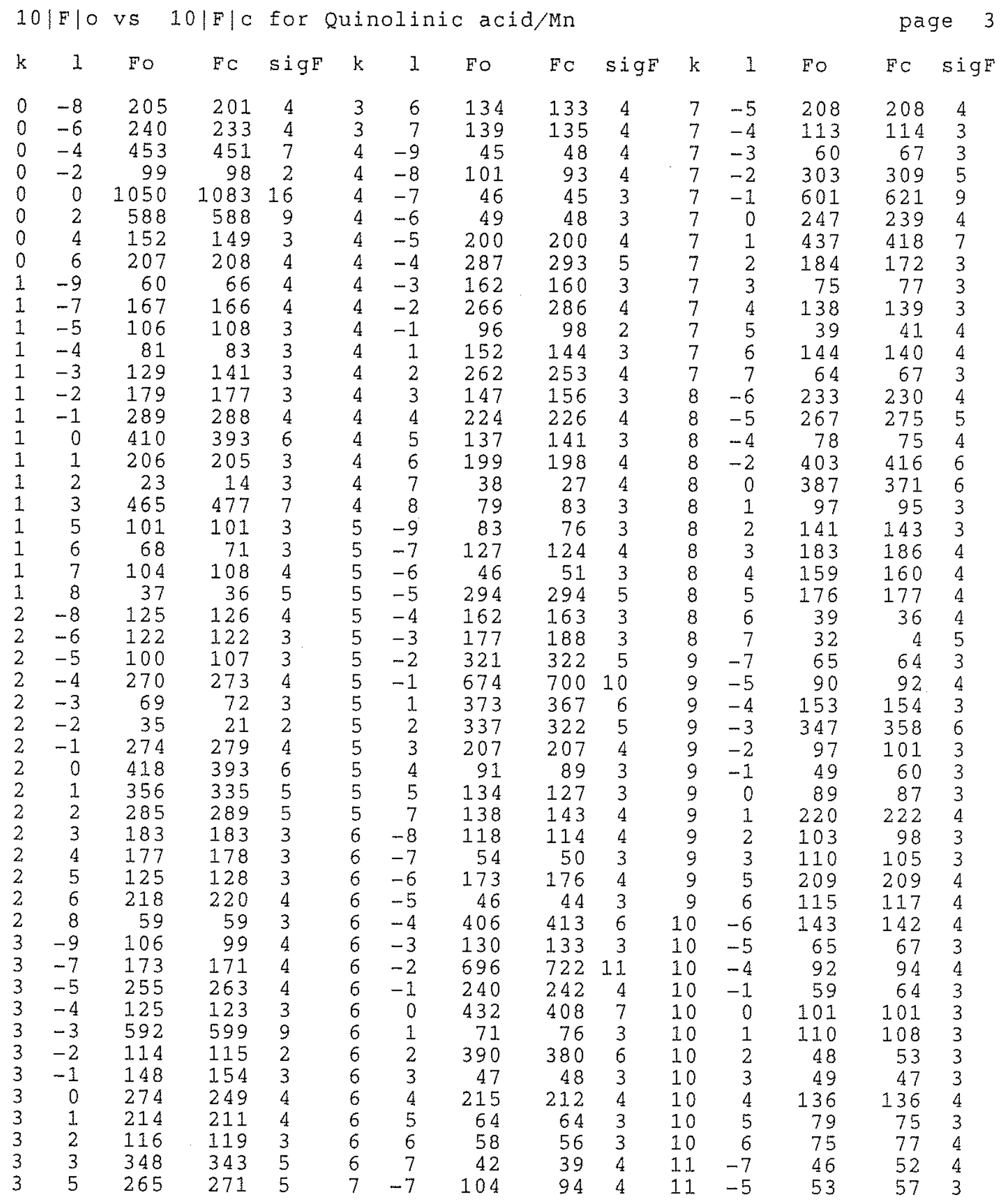




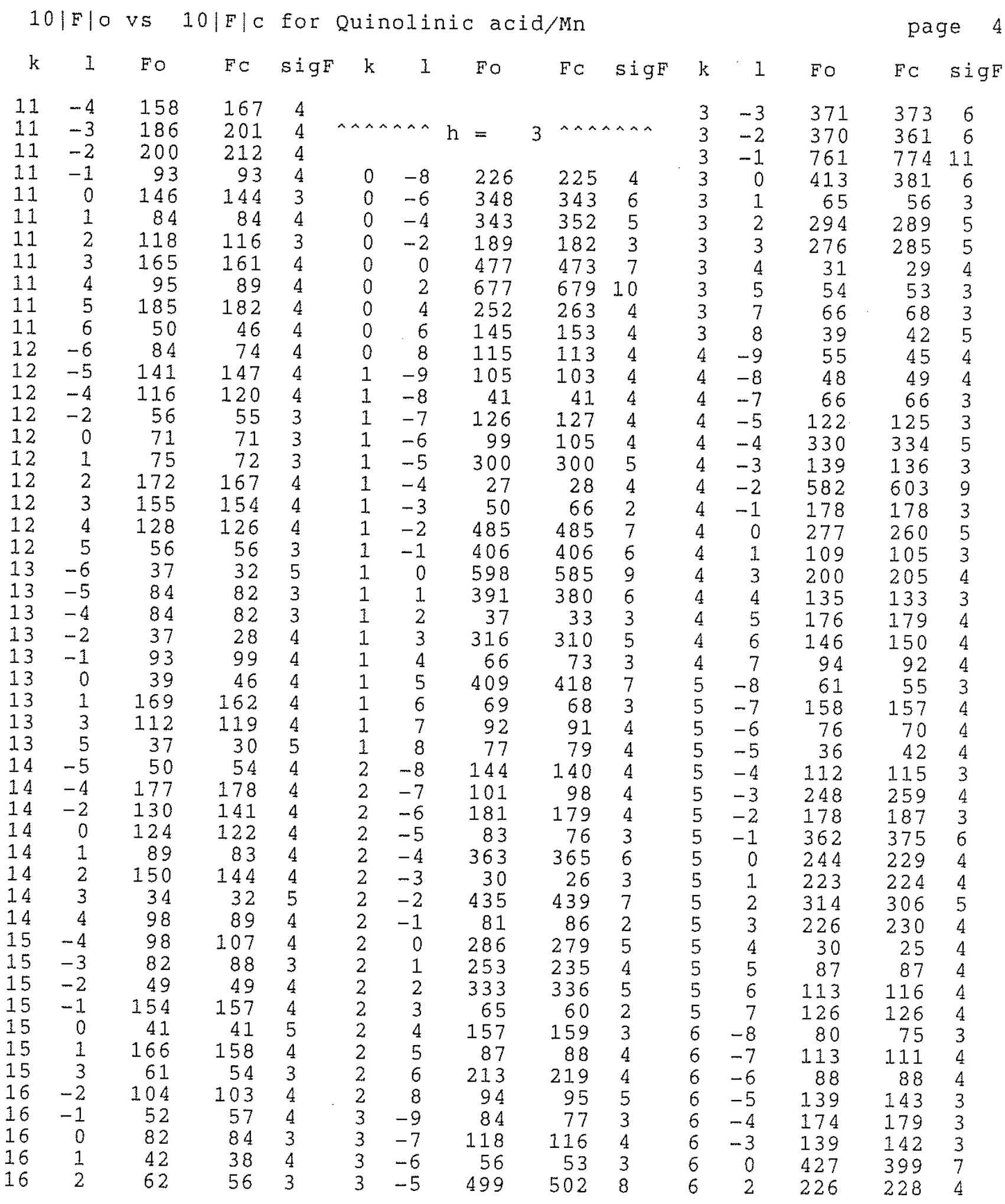




\begin{tabular}{|c|c|c|c|c|c|c|c|c|c|c|c|c|c|c|}
\hline 1 & Fo & $\mathrm{FC}$ & $\operatorname{sigF}$ & k & 1 & EO & & FC & $\operatorname{sigF}$ & k & 1 & Fo & $\mathrm{FC}$ & $\operatorname{sigF}$ \\
\hline 3 & 86 & 86 & 3 & 10 & 3 & 51 & & 51 & 3 & 0 & -6 & 582 & 581 & 9 \\
\hline 4 & 188 & 187 & 4 & 10 & 4 & 102 & & 105 & 4 & 0 & -4 & 130 & 1.33 & 3 \\
\hline 5 & 79 & 78 & 3 & 10 & 5 & 54 & & 58 & 3 & 0 & -2 & 618 & 621 & 9 \\
\hline-7 & 145 & 146 & 4 & 10 & 6 & 146 & & 142 & 4 & 0 & 0 & 97 & 86 & 3 \\
\hline-6 & 37 & 31 & 4 & 11 & -7 & 73 & & 74 & 3 & 0 & 2 & 491 & 511 & 8 \\
\hline-5 & 184 & 187 & 4 & 11 & -6 & 41 & & 51 & 4 & 0 & 4 & 223 & 237 & 4 \\
\hline-4 & 54 & 59 & 3 & 11 & -5 & 248 & & 259 & 5 & 0 & 6 & 111 & 114 & 4 \\
\hline-3 & 154 & 160 & 3 & 11 & -3 & 280 & & 299 & 5 & 1 & -9 & 95 & 92 & 4 \\
\hline-2 & 153 & 143 & 3 & 11 & -2 & 56 & & 68 & 3 & 1 & -8 & 1.22 & 119 & 4 \\
\hline-1 & 31 & 25 & 3 & 11 & -1 & 186 & & 191 & 4 & 1 & -7 & 116 & 119 & 4 \\
\hline 0 & 117 & 109 & 3 & 11 & 0 & 86 & & 79 & 4 & 1 & -6 & 204 & 205 & 4 \\
\hline 1 & 509 & 494 & 8 & 11 & 1 & 98 & & 99 & 4 & 1 & -5 & 374 & 379 & 6 \\
\hline 2 & 137 & 137 & 3 & 11 & 2 & 58 & & 61 & 3 & 1 & -3 & 466 & 464 & 7 \\
\hline 3 & 165 & 169 & 3 & 11 & 3 & 87 & & 86 & 4 & 1 & -2 & 183 & 177 & 3 \\
\hline 4 & 69 & 69 & 3 & 11 & 4 & 50 & & 48 & 3 & 1 & -1 & 490 & 494 & 7 \\
\hline 5 & 91 & 89 & 4 & 11 & 5 & 151 & & 153 & 4 & 1 & 0 & 372 & 363 & 6 \\
\hline 7 & 100 & 101 & 4 & 12 & -6 & 130 & & 136 & 4 & 1 & 1 & 152 & 127 & 3 \\
\hline-8 & 93 & 94 & 5 & 12 & -5 & 132 & & 139 & 4 & 1 & 2 & 46 & 49 & 2 \\
\hline-6 & 170 & 168 & 4 & 12 & -4 & 186 & & 193 & 4 & 1 & 3 & 301 & 311 & 5 \\
\hline-5 & 207 & 207 & 4 & 12 & -2 & 158 & & 166 & 4 & 1 & 4 & 256 & 264 & 4 \\
\hline-4 & 28 & 27 & 5 & 12 & 0 & 123 & & 121 & 4 & 1 & 5 & 133 & 134 & 3 \\
\hline-2 & 118 & 133 & 3 & 12 & 1 & 76 & & 73 & 3 & 1 & 6 & 89 & 88 & 4 \\
\hline-1 & 86 & 87 & 3 & 12 & 3 & 35 & & 36 & 4 & 1 & 7 & 95 & 96 & 4 \\
\hline 0 & 289 & 273 & 5 & 12 & 4 & 172 & & 163 & 4 & 2 & -9 & 84 & 79 & 4 \\
\hline 1 & 69 & 60 & 3 & 13 & -6 & 43 & & 38 & 4 & 2 & -8 & 111 & 109 & 4 \\
\hline 2 & 377 & 373 & 6 & 13 & -5 & 80 & & 76 & 3 & 2 & -7 & 139 & 143 & 4 \\
\hline 3 & 73 & 72 & 4 & 13 & -2 & 118 & & 127 & 4 & 2 & -6 & 136 & 133 & 3 \\
\hline 4 & 118 & 119 & 4 & 13 & -1 & 117 & & 121 & 4 & 2 & -5 & 178 & 184 & 4 \\
\hline 5 & 92 & 87 & 4 & 13 & 0 & 36 & & 36 & 4 & 2 & -4 & 293 & 297 & 5 \\
\hline 6 & 79 & 73 & 3 & 13 & 1 & 75 & & 68 & 3 & 2 & -3 & 311 & 305 & 5 \\
\hline-8 & 48 & 49 & 4 & 13 & 3 & 77 & & 80 & 3 & 2 & -2 & 457 & 476 & 7 \\
\hline-7 & 90 & 81 & 4 & 14 & -4 & 80 & & 83 & 3 & 2 & -1 & 39 & 42 & 2 \\
\hline-5 & 174 & 180 & 4 & 14 & -3 & 84 & & 90 & 4 & 2 & 0 & 252 & 246 & 4 \\
\hline-3 & 333 & 349 & 5 & 14 & 0 & 87 & & 77 & 4 & 2 & 1 & 342 & 325 & 5 \\
\hline-2 & 134 & 135 & 3 & 14 & 1 & 40 & & 44 & 5 & 2 & 2 & 300 & 308 & 5 \\
\hline-1 & 85 & 86 & 3 & 14 & 2 & 62 & & 60 & 3 & 2 & 3 & 33 & 27 & 3 \\
\hline 0 & 175 & 170 & 4 & 14 & 3 & 69 & & 66 & 4 & 2 & 4 & 108 & 108 & 3 \\
\hline 1 & 341 & 321 & 6 & 14 & 4 & 127 & & 122 & 4 & 2 & 5 & 323 & 333 & 5 \\
\hline 2 & 119 & 118 & 3 & 15 & -2 & 99 & & 109 & 4 & 2 & 6 & 170 & 1.75 & 4 \\
\hline 3 & 135 & 131 & 3 & 15 & -1 & 68 & & 63 & 4 & 2 & 7 & 101 & 88 & 4 \\
\hline 4 & 37 & 34 & 4 & 15 & 0 & 49 & & 45 & 4 & 3 & -9 & 62 & 58 & 3 \\
\hline 5 & 203 & 206 & 4 & 15 & 1 & 102 & & 92 & 5 & 3 & -8 & 79 & 82 & 3 \\
\hline 6 & 71 & 71 & 3 & 15 & 3 & 71 & & 73 & 3 & 3 & -7 & 148 & 139 & 4 \\
\hline-6 & 154 & 163 & 4 & 16 & -2 & 57 & & 58 & 4 & 3 & -6 & 308 & 314 & 5 \\
\hline-5 & 79 & 77 & 4 & 1.6 & -1 & 37 & & 38 & 5 & 3 & -5 & 146 & 147 & 3 \\
\hline-4 & 116 & 112 & 3 & 16 & 0 & 64 & & 61 & 3 & 3 & -4 & 57 & 53 & 2 \\
\hline-3 & 99 & 102 & 4 & & & & & & & 3 & -3 & 113 & 125 & 3 \\
\hline-2 & 369 & 384 & 6 & ^^^ & $\leadsto h$ & $=$ & 4 & $\wedge \wedge$ & $\cdots$ & 3 & -2 & 68 & 66 & 3 \\
\hline 0 & 149 & 147 & 4 & & & & & & & 3 & -1 & 454 & 456 & 7 \\
\hline 2 & 151 & 154 & 4 & 0 & -8 & 156 & & 152 & 4 & 3 & 0 & 243 & 230 & 4 \\
\hline
\end{tabular}




\begin{tabular}{|c|c|c|c|c|c|c|c|c|c|c|c|c|c|}
\hline 1 & Fo & $\mathrm{FC}$ & sigF & k & 1 & Fo & EC & $\operatorname{sigF}$ & $k$ & 1 & Fo & $\mathrm{FC}$ & $\mathrm{sigF}$ \\
\hline 1 & 319 & 312 & 5 & 7 & -7 & 110 & 110 & 4 & 11 & 1 & 112 & 109 & 4 \\
\hline 3 & 183 & 188 & 4 & 7 & -6 & 138 & 140 & 4 & 11 & 2 & 55 & 51 & 3 \\
\hline 4 & 310 & 317 & 5 & 7 & -5 & 218 & 218 & 4 & 11 & 3 & 84 & 81 & 4 \\
\hline 5 & 71 & 71 & 3 & 7 & -4 & 105 & 113 & 3 & 11 & 5 & 56 & 46 & 3 \\
\hline 6 & 75 & 69 & 3 & 7 & -3 & 162 & 172 & 3 & 12 & -6 & 94 & 100 & 3 \\
\hline 7 & 95 & 92 & 4 & 7 & -1 & 1.40 & 145 & 3 & 12 & -5 & 68 & 68 & 4 \\
\hline-9 & 44 & 37 & 4 & 7 & 0 & 80 & 76 & 3 & 12 & -4 & 104 & 114 & 4 \\
\hline-8 & 93 & 89 & 4 & 7 & 1 & 328 & 326 & 5 & 12 & -2 & 132 & 129 & 4 \\
\hline-7 & 53 & 50 & 3 & 7 & 2 & 52 & 52 & 3 & 12 & 0 & 172 & 165 & 4 \\
\hline-6 & 175 & 175 & 4 & 7 & 3 & 168 & 174 & 4 & 12 & 2 & 39 & 38 & 4 \\
\hline-5 & 56 & 54 & 3 & 7 & 4 & 129 & 133 & 4 & 12 & 3 & 59 & 57 & 3 \\
\hline-4 & 332 & 337 & 5 & 7 & 5 & 200 & 206 & 4 & 12 & 4 & 69 & 67 & 3 \\
\hline-3 & 136 & 149 & 3 & 8 & -8 & 110 & 105 & 4 & 13 & -5 & 71 & 67 & 4 \\
\hline-2 & 162 & 186 & 3 & 8 & -6 & 246 & 248 & 5 & 13 & -3 & 162 & 175 & 4 \\
\hline-1 & 182 & 177 & 3 & 8 & -4 & 128 & 133 & 3 & 13 & -1 & 79 & 79 & 3 \\
\hline 0 & 448 & 423 & 7 & 8 & -3 & 74 & 80 & 3 & 13 & 0 & 46 & 46 & 4 \\
\hline 1 & 53 & 48 & 3 & 8 & -2 & 153 & 155 & 3 & 13 & 1 & 124 & 11.4 & 4 \\
\hline 2 & 195 & 190 & 4 & 8 & -1 & 40 & 41 & 3 & 13 & 3 & 83 & 77 & 4 \\
\hline 3 & 112 & 114 & 3 & 8 & 0 & 236 & 229 & 4 & 14 & -4 & 108 & 117 & 4 \\
\hline 4 & 380 & 387 & 6 & 8 & 1 & 93 & 90 & 4 & 14 & -3 & 61 & 61 & 3 \\
\hline 5 & 51 & 52 & 3 & 8 & 2 & 179 & 177 & 4 & 14 & -2 & 96 & 103 & 4 \\
\hline 6 & 143 & 151 & 4 & 8 & 3 & 33 & 32 & 4 & 14 & 0 & 82 & 72 & 3 \\
\hline-9 & 95 & 79 & 4 & 8 & 4 & 112 & 106 & 3 & 14 & 1 & 53 & 48 & 4 \\
\hline-8 & 68 & 68 & 3 & 8 & 6 & 54 & 48 & 3 & 14 & 2 & 49 & 46 & 4 \\
\hline-7 & 12,4 & 123 & 4 & 9 & -8 & 57 & 52 & 4 & 14 & 3 & 35 & 33 & 5 \\
\hline-5 & 120 & 120 & 3 & 9 & -7 & 93 & 94 & 4 & 15 & -3 & 55 & 52 & 4 \\
\hline-4 & 152 & 149 & 3 & 9 & -6 & 60 & 63 & 3 & 15 & -2 & 36 & 48 & 5 \\
\hline-2 & 329 & 321 & 5 & 9 & -5 & 124 & 127 & 4 & 15 & -1 & 74 & 74 & 3 \\
\hline-1 & 260 & 265 & 4 & 9 & -3 & 218 & 231 & 4 & 15 & 1 & 81 & 68 & 3 \\
\hline 0 & 138 & 1.34 & 3 & 9 & -2 & 116 & 1.09 & 3 & 16 & -2 & 93 & 98 & 5 \\
\hline 1 & 552 & 539 & 9 & 9 & -1 & 141 & 140 & 3 & & & & & \\
\hline 3 & 141 & 148 & 3 & 9 & 0 & 107 & 104 & 4 & $\wedge \wedge \wedge$ & $\cdots h$ & $=$ & $\wedge \hat{\mu}$ & $\wedge$ \\
\hline 4 & 52 & 56 & 3 & 9 & 1 & 97 & 102 & 4 & & & & & \\
\hline 5 & 199 & 205 & 4 & 9 & 3 & 137 & 138 & 4 & 0 & -8 & 171 & 168 & 4 \\
\hline 7 & 64 & 61 & 3 & 9 & 5 & 62 & 61 & 3 & 0 & -6 & 29 & 30 & 5 \\
\hline-8 & 154 & 140 & 4 & 10 & -7 & 45 & 39 & 4 & 0 & -4 & 54 & 65 & 2 \\
\hline-7 & 90 & 92 & 4 & 10 & -6 & 72 & 74 & 3 & 0 & -2 & 143 & 142 & 3 \\
\hline-6 & 177 & 182 & 4 & 10 & -5 & 32 & 29 & 5 & 0 & 0 & 265 & 257 & 4 \\
\hline-5 & 37 & 42 & 4 & 10 & -4 & 120 & 124 & 3 & 0 & 2 & 378 & 379 & 6 \\
\hline-4 & 170 & 176 & 3 & 10 & -3 & 41 & 35 & 4 & 0 & 4 & 129 & 131 & 3 \\
\hline-3 & 142 & 144 & 3 & 10 & -2 & 153 & 156 & 4 & 0 & 6 & 74 & 76 & 3 \\
\hline-2 & 100 & 107 & 3 & 10 & 0 & 251 & 236 & 5 & 1 & -9 & 83 & 85 & 3 \\
\hline-1 & 106 & 108 & 3 & 10 & 1 & 33 & 34 & 5 & 1 & -8 & 110 & 109 & 4 \\
\hline 0 & 266 & 249 & 5 & 10 & 2 & 59 & 59 & 3 & 1 & -7 & 111 & 110 & 4 \\
\hline 1 & 38 & 32 & 4 & 10 & 4 & 119 & 125 & 4 & 1 & -6 & 37 & 31 & 4 \\
\hline 2 & 266 & 264 & 5 & 11 & -7 & 65 & 65 & 3 & 1 & -5 & 334 & 333 & 5 \\
\hline 3 & 78 & 85 & 4 & 11 & -5 & 39 & 37 & 4 & 1 & -2 & 80 & 80 & 3 \\
\hline 4 & 191 & 188 & 4 & 11 & -3 & 229 & 245 & 4 & 1 & -1 & 431 & 424 & 7 \\
\hline 5 & 107 & 106 & 4 & 11 & -1 & 116 & 119 & 4 & 1 & 0 & 502 & 483 & 8 \\
\hline 7 & 61 & 55 & 3 & 11 & 0 & 60 & 58 & 3 & 1 & 1 & 346 & 353 & 6 \\
\hline
\end{tabular}




\begin{tabular}{|c|c|c|c|c|c|c|c|c|c|c|c|c|c|}
\hline 1 & FO & $\mathrm{FC}$ & sigF & $k$ & 1 & Fo & $\mathrm{FC}$ & sigF & $\mathrm{k}$ & 1 & Fo & FC & $\operatorname{sigF}$ \\
\hline 3 & 157 & 158 & 3 & 5 & -7 & 293 & 290 & 5 & 9 & -6 & 48 & 38 & 4 \\
\hline 4 & 197 & 205 & 4 & 5 & -6 & 50 & 45 & 3 & 9 & -5 & 69 & 65 & 3 \\
\hline 5 & 218 & 224 & 4 & 5 & -5 & 172 & 178 & 4 & 9 & -4 & 88 & 94 & 4 \\
\hline-8 & 92 & 94 & 4 & 5 & -3 & 297 & 300 & 5 & 9 & -3 & 137 & 139 & 4 \\
\hline-6 & 130 & 132 & 4 & 5 & -2 & 146 & 153 & 3 & 9 & -2 & 34 & 28 & 4 \\
\hline-5 & 43 & 48 & 3 & 5 & -1 & 200 & 200 & 4 & 9 & -1 & 170 & 162 & 4 \\
\hline-4 & 234 & 234 & 4 & 5 & 0 & 163 & 156 & 3 & 9 & 1 & 55 & 43 & 3 \\
\hline-3 & 455 & 445 & 7 & 5 & 1 & 229 & 231 & 4 & 9 & 2 & 45 & 44 & 4 \\
\hline-2 & 430 & 441 & 7 & 5 & 3 & 288 & 295 & 5 & 9 & 3 & 62 & 64 & 3 \\
\hline-1 & 48 & 39 & 2 & 5 & 4 & 133 & 131 & 4 & 9 & 5 & 58 & 64 & 3 \\
\hline 0 & 146 & 147 & 3 & 5 & 5 & 78 & 81 & 3 & 10 & -7 & 61 & 62 & 3 \\
\hline 1 & 184 & 177 & 4 & 6 & -8 & 144 & 141 & 4 & 10 & -6 & 99 & 98 & 4 \\
\hline 2 & 331 & 338 & 5 & 6 & -6 & 173 & 173 & 4 & 10 & -4 & 125 & 132 & 4 \\
\hline 3 & 255 & 261. & 4 & 6 & -5 & 45 & 46 & 3 & 10 & -2 & 149 & 152 & 4 \\
\hline 4 & 61 & 63 & 3 & 6 & -4 & 174 & 172 & 4 & 10 & -1 & 37 & 36 & 4 \\
\hline 5 & 93 & 90 & 4 & 6 & -3 & 110 & 108 & 3 & 10 & 0 & 146 & 132 & 4 \\
\hline 6 & 95 & 104 & 4 & 6 & -2 & 94 & 93 & 3 & 10 & 1 & 61 & 60 & 3 \\
\hline-9 & 143 & 128 & 4 & 6 & -1 & 85 & 88 & 3 & 10 & 2 & 99 & 100 & 4 \\
\hline-8 & 98 & 91 & 4 & 6 & 0 & 317 & 300 & 5 & 10 & 3 & 65 & 67 & 3 \\
\hline-7 & 201 & 199 & 4 & 6 & 1 & 120 & 117 & 4 & 10 & 4 & 62 & 63 & 3 \\
\hline-6 & 67 & 62 & 3 & 6 & 2 & 115 & 113 & 4 & 11 & -7 & 37 & 37 & 5 \\
\hline-5 & 142 & 144 & 3 & 6 & 3 & 106 & 98 & 3 & 11 & -5 & 51 & 53 & 3 \\
\hline-4 & 74 & 74 & 3 & 6 & 4 & 226 & 232 & 4 & 11 & -4 & 33 & 35 & 5 \\
\hline-3 & 363 & 367 & 6 & 6 & 6 & 99 & 102 & 4 & 11 & -3 & 1.76 & 184 & 4 \\
\hline-2 & 39 & 42 & 3 & 7 & -7 & 151 & 152 & 4 & 11 & -1 & 137 & 140 & 4 \\
\hline-1 & 271 & 269 & 4 & 7 & -5 & 184 & 184 & 4 & 11 & 0 & 55 & 47 & 4 \\
\hline 0 & 452 & 422 & 7 & 7 & -4 & 174 & 186 & 4 & 11 & 2 & 40 & 33 & 4 \\
\hline 1 & 198 & 203 & 4 & 7 & -3 & 108 & 109 & 3 & 11 & 4 & 39 & 37 & 4 \\
\hline 3 & 72 & 69 & 4 & 7 & -1 & 193 & 194 & 4 & 12 & -5 & 52 & 55 & 4 \\
\hline 4 & 154 & 156 & 4 & 7 & 0 & 69 & 63 & 3 & 12 & -4 & 111 & 110 & 4 \\
\hline 5 & 95 & 101 & 4 & 7 & 1 & 226 & 218 & 4 & 12 & -3 & 109 & 113 & 4 \\
\hline 7 & 65 & 67 & 3 & 7 & 2 & 49 & 50 & 3 & 12 & -2 & 114 & 118 & 4 \\
\hline-9 & 49 & 48 & 4 & 7 & 3 & 416 & 420 & 7 & 12 & 0 & 107 & 100 & 4 \\
\hline-8 & 78 & 76 & 3 & 7 & 4 & 66 & 63 & 3 & 1.2 & 2 & 34 & 35 & 5 \\
\hline-7 & 85 & 78 & 4 & 7 & 5 & 111 & 107 & 4 & 13 & -5 & 62 & 64 & 3 \\
\hline-6 & 256 & 258 & 5 & 8 & -8 & 81 & 81 & 4 & 13 & -3 & 123 & 126 & 4 \\
\hline-5 & 135 & 135 & 3 & 8 & -7 & 106 & 111 & 4 & 13 & -1 & 75 & 74 & 3 \\
\hline-4 & 314 & 318 & 5 & 8 & -6 & 139 & 137 & 4 & 13 & 0 & 49 & 42 & 4 \\
\hline-3 & 58 & 58 & 3 & 8 & -5 & 86 & 88 & 4 & 13 & 1 & 182 & 174 & 5 \\
\hline-2 & 477 & 484 & 7 & 8 & -4 & 105 & 113 & 3 & 13 & 3 & 94 & 90 & 4 \\
\hline-1 & 176 & 173 & 3 & 8 & -3 & 43 & 48 & 3 & 14 & -4 & 82 & 88 & 3 \\
\hline 0 & 139 & 140 & 3 & 8 & -2 & 41 & 38 & 3 & 14 & -3 & 45 & 47 & 4 \\
\hline 1 & 37 & 39 & 4 & 8 & 0 & 296 & 278 & 5 & 14 & -2 & 87 & 87 & 3 \\
\hline 2 & 195 & 198 & 4 & 8 & 1 & 47 & 46 & 4 & 14 & -1 & 57 & 50 & 3 \\
\hline 3 & 123 & 134 & 3 & 8 & 2 & 142 & 144 & 4 & 14 & 0 & 141 & 133 & 4 \\
\hline 4 & 258 & 271 & 5 & 8 & 3 & 59 & 59 & 3 & 14 & 1 & 120 & 110 & 5 \\
\hline 6 & 136 & 137 & 4 & 8 & 4 & 159 & 155 & 4 & 14 & 2 & 72 & 66 & 3 \\
\hline 7 & 34 & 23 & 5 & 8 & 5 & 31 & 33 & 5 & 15 & -3 & 75 & 75 & 4 \\
\hline-9 & 74 & 71 & 4 & 8 & 6 & 48 & 47 & 4 & 15 & -2 & 55 & 64 & 4 \\
\hline-8 & 61 & 59 & & 9 & -7 & 57 & 53 & 3 & 15 & -1 & 54 & 52 & \\
\hline
\end{tabular}




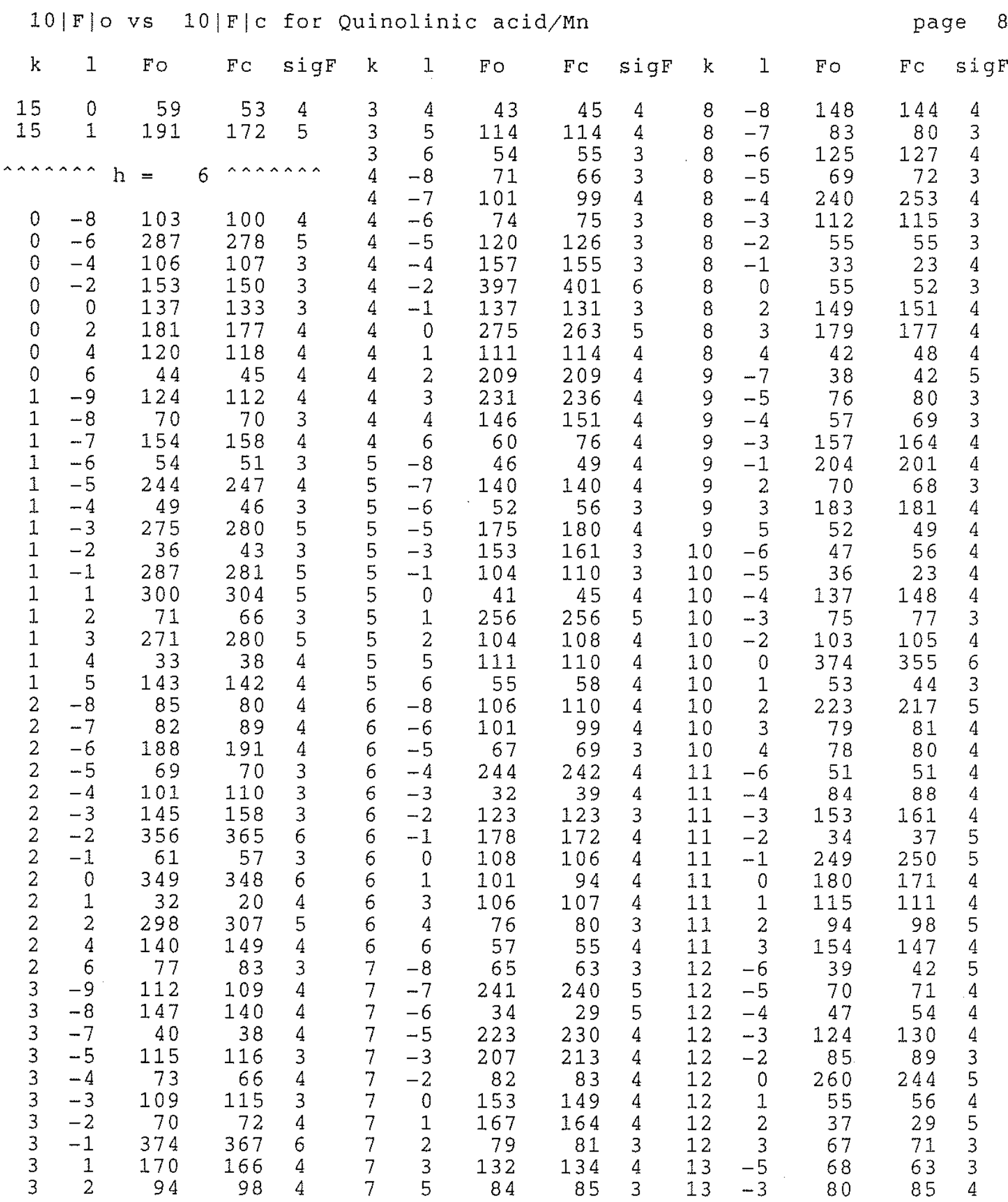




$$
\begin{aligned}
& \text { 10|F|O vs } 10|\mathrm{~F}| \mathrm{C} \text { for Quinolinic acid/Mn } \\
& \text { page } 9 \\
& k 1 \text { FO FC sigF k } 1 \text { FO FC sigF k } 1 \text { Fo FC sigF }
\end{aligned}
$$

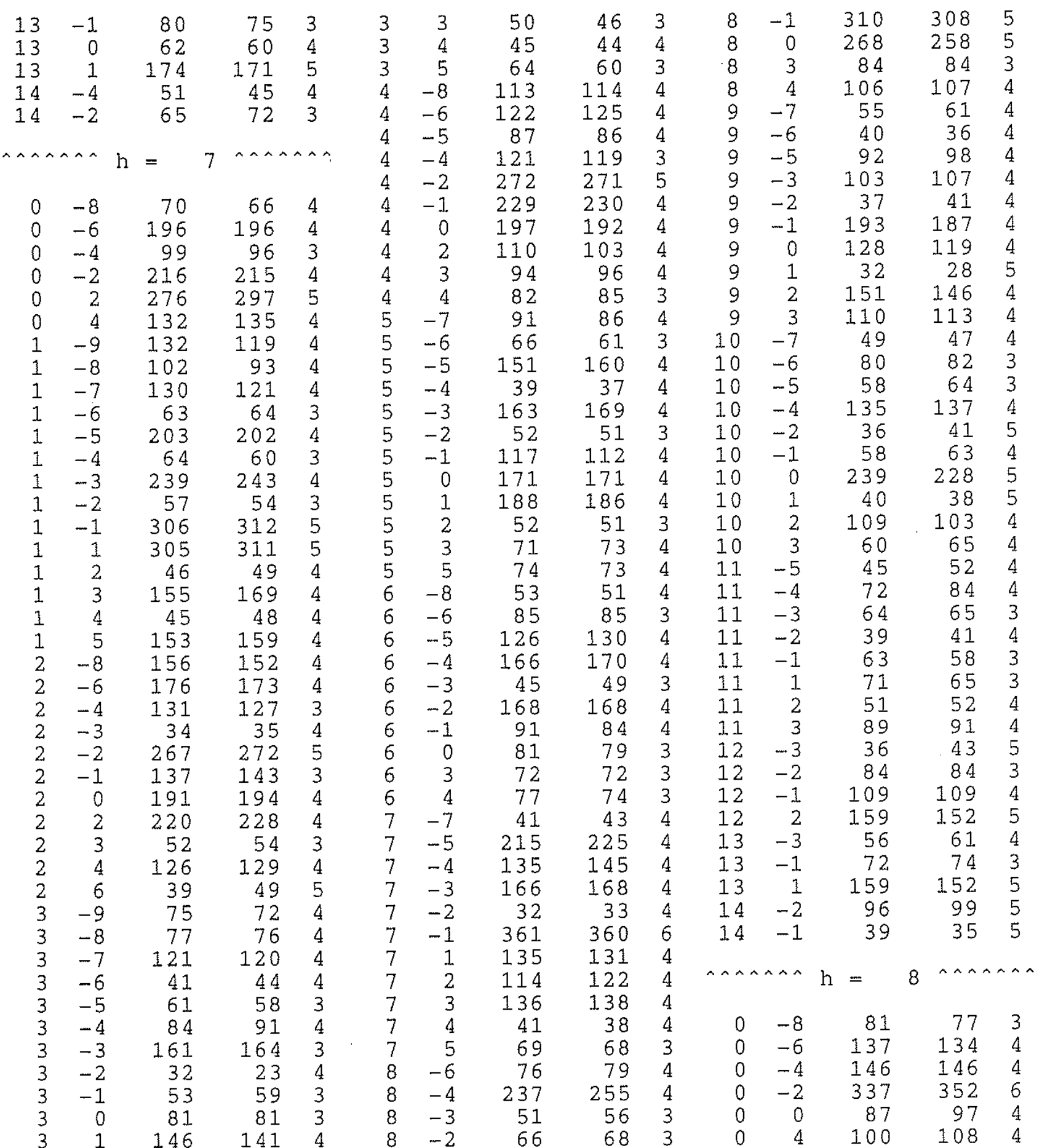


$10|\mathrm{~F}| 0$ vs $10|\mathrm{~F}| \mathrm{C}$ for Quinolinic acid/Mn

page 10

\begin{tabular}{|c|c|c|c|c|c|c|c|c|c|c|c|c|c|c|c|}
\hline 1 & Fo & $\mathrm{FC}$ & $\operatorname{sigF}$ & $\mathrm{k}$ & 1 & Fo & $\mathrm{FC}$ & $\mathrm{SIgF}$ & $\mathrm{k}$ & 1 & & FO & & $\mathrm{F}^{\prime} \mathrm{C}$ & sig \\
\hline-7 & 139 & 133 & 4 & 5 & -3 & 112 & 114 & 4 & 11 & -2 & & 39 & & 33 & 5 \\
\hline-6 & 64 & 65 & 3 & 5 & -2 & 193 & 197 & 4 & 11 & -1 & & 98 & & 93 & 5 \\
\hline-5 & 82 & 73 & 4 & 5 & -1 & 341 & 345 & 6 & 12 & -4 & & 80 & & 85 & 4 \\
\hline-4 & 69 & 69 & 3 & 5 & 0 & 60 & 61 & 3 & 12 & -3 & & 56 & & 57 & 4 \\
\hline-3 & 271 & 268 & 5 & 5 & 1 & 180 & 180 & 4 & 12 & 0 & & 65 & & 62 & 4 \\
\hline-2 & 108 & 104 & 4 & 5 & 2 & 92 & 101 & 4 & 13 & -3 & & 54 & & 48 & 4 \\
\hline-1 & 52 & 57 & 3 & 5 & 3 & 1.27 & 132 & 4 & 13 & -1 & & 78 & & 75 & 4 \\
\hline 1 & 146 & 142 & 4 & 6 & -8 & 81 & 80 & 4 & 13 & 0 & & 45 & & 46 & \\
\hline 2 & 98 & 103 & 4 & 6 & -6 & 50 & 55 & 4 & & & & & & & \\
\hline 3 & 49 & 65 & 4 & 6 & -4 & 126 & 132 & 4 & A & $\cdots$ & $\mathrm{h}=$ & $=$ & 9 & $\wedge \wedge \wedge$ & \\
\hline 5 & 139 & 142 & 4 & 6 & -2 & 196 & 191 & 4 & & & & & & & \\
\hline-8 & 70 & 68 & 3 & 6 & -1 & 164 & 161 & 4 & 0 & -6 & & 265 & & 276 & 5 \\
\hline-6 & 167 & 174 & 4 & 6 & 0 & 63 & 55 & 3 & 0 & -4 & & 111 & & 115 & 4 \\
\hline-5 & 84 & 86 & 4 & 6 & 2 & 141 & 145 & 4 & 0 & -2 & & 224 & & 220 & 4 \\
\hline-4 & 127 & 128 & 4 & 6 & 4 & 35 & 37 & 5 & 0 & 0 & & 107 & & 117 & 4 \\
\hline-2 & 153 & 159 & 4 & 7 & -7 & 108 & 105 & 4 & 0 & 2 & & 63 & & 57 & 3 \\
\hline-1 & 126 & 130 & 4 & 7 & -5 & 127 & 124 & 4 & 0 & 4 & & 140 & & 144 & 4 \\
\hline 0 & 198 & 204 & 4 & 7 & -3 & 148 & 148 & 4 & 1 & -7 & & 140 & & 137 & 4 \\
\hline 1 & 47 & 45 & 3 & 7 & -2 & 32 & 19 & 5 & 1 & -6 & & 58 & & 63 & 3 \\
\hline 2 & 92 & 95 & 4 & 7 & -1 & 211 & 211 & 4 & 1 & -5 & & 159 & & 161 & 4 \\
\hline 4 & 124 & 135 & 4 & 7 & 0 & 32 & 35 & 5 & 1 & -4 & & 85 & & 86 & 4 \\
\hline 5 & 148 & 145 & 4 & 7 & 1 & 86 & 90 & 4 & 1 & -3 & & 348 & & 351 & 6 \\
\hline-7 & 59 & 55 & 3 & 7 & 2 & 45 & 47 & 4 & 1 & -2 & & 203 & & 205 & 4 \\
\hline-6 & 88 & 86 & 4 & 7 & 3 & 120 & 119 & 4 & 1 & -1 & & 149 & & 146 & 4 \\
\hline-5 & 140 & 146 & 4 & 8 & -6 & 66 & 67 & 3 & 1 & 0 & & 52 & & 48 & 3 \\
\hline-4 & 36 & 36 & 4 & 8 & -5 & 31 & 29 & 5 & 1 & 1 & & 103 & & 97 & 4 \\
\hline-3 & 116 & 122 & 4 & 8 & -4 & 114 & 111 & 4 & 1 & 3 & & 74 & & 87 & 4 \\
\hline-1 & 147 & 148 & 4 & 8 & -2 & 43 & 40 & 4 & 1 & 4 & & 94 & & 95 & 4 \\
\hline 1 & 219 & 221 & 4 & 8 & -1 & 46 & 48 & 4 & 2 & -8 & & 73 & & 66 & 3 \\
\hline 2 & 63 & 65 & 3 & 8 & 0 & 88 & 86 & 5 & 2 & -6 & & 148 & & 151 & 4 \\
\hline 3 & 72 & 70 & 4 & 8 & 1 & 63 & 65 & 3 & 2 & -5 & & 109 & & 116 & 4 \\
\hline 4 & 42 & 29 & 4 & 8 & 2 & 127 & 125 & 4 & 2 & -4 & & 132 & & 136 & 4 \\
\hline 5 & 59 & 54 & 4 & 8 & 4 & 50 & 44 & 4 & 2 & -3 & & 197 & & 202 & 4 \\
\hline-8 & 64 & 66 & 3 & 9 & -7 & 61 & 60 & 4 & 2 & -2 & & 167 & & 162 & 4 \\
\hline-7 & 47 & 45 & 4 & 9 & -6 & 33 & 32 & 5 & 2 & 0 & & 123 & & 123 & 4 \\
\hline-6 & 139 & 142 & 4 & 9 & -4 & 46 & 47 & 4 & 2 & 1 & & 169 & & 1.88 & 4 \\
\hline-5 & 1.12 & 128 & 4 & 9 & -3 & 96 & 96 & 4 & 2 & 2 & & 79 & & 80 & 3 \\
\hline-4 & 114 & 124 & 4 & 9 & -2 & 56 & 60 & 3 & 2 & 4 & & 124 & & 119 & 4 \\
\hline-3 & 38 & 31 & 4 & 9 & -1 & 58 & 49 & 4 & 3 & -7 & & 85 & & 74 & 4 \\
\hline-2 & 536 & 543 & 8 & 9 & 1 & 56 & 49 & 3 & 3 & -6 & & 74 & & 78 & 3 \\
\hline-1 & 111 & 110 & 4 & 9 & 3 & 63 & 62 & 4 & 3 & -5 & & 51 & & 51 & 3 \\
\hline 0 & 205 & 200 & 4 & 10 & -6 & 69 & 67 & 3 & 3 & -4 & & 76 & & 78 & 3 \\
\hline 1 & 73 & 68 & 3 & 10 & -4 & 93 & 96 & 4 & 3 & -3 & & 50 & & 45 & 3 \\
\hline 2 & 170 & 174 & 4 & 10 & -2 & 96 & 89 & 4 & 3 & -2 & & 270 & & 272 & 5 \\
\hline 3 & . 52 & 49 & 4 & 10 & -1 & 70 & 69 & 3 & 3 & -1 & & 90 & & 92 & 4 \\
\hline 4 & 104 & 108 & 4 & 10 & 0 & 141 & 137 & 4 & 3 & 1 & & 115 & & 117 & 4 \\
\hline 5 & 34 & 30 & 6 & 10 & 1 & 46 & 44 & 4 & 3 & 2 & & 58 & & 61 & 3 \\
\hline-7 & 85 & 81 & 4 & 10 & 2 & 81 & 76 & 4 & 3 & 3 & & 72 & & 65 & 3 \\
\hline-5 & 216 & 229 & 4 & 11 & -5 & 56 & 53 & 4 & 3 & 4 & & 83 & & 75 & 4 \\
\hline-4 & 61 & 62 & 3 & 11 & -3 & 56 & 55 & 3 & 4 & -8 & & 140 & & 129 & \\
\hline
\end{tabular}


$10|\mathrm{~F}| 0$ vs $10|\mathrm{~F}| \mathrm{C}$ for Quinolinic acid/Mn

page 11

\begin{tabular}{|c|c|c|c|c|c|c|c|c|c|c|c|c|c|}
\hline 1 & Fo & $\mathrm{FC}$ & $\operatorname{sig} F$ & $\mathrm{k}$ & 1 & FO & $\mathrm{FC}$ & sigF & $\mathrm{k}$ & 1 & Fo & $\mathrm{FC}$ & sigF \\
\hline-4 & 111 & 109 & 4 & 12 & -2 & 59 & 53 & 4 & 5 & 1 & 69 & 68 & 3 \\
\hline-3 & 112 & 108 & 4 & & & & & & 6 & -3 & 91 & 93 & 4 \\
\hline-2 & 78 & 76 & 3 & $\wedge \wedge \wedge$ & $\hat{x}$ & $\mathrm{~h}=$ & $\leadsto \wedge \wedge$ & ^^ & 6 & -2 & 88 & 89 & 3 \\
\hline 0 & 152 & 147 & 4 & & & & & & 6 & 1 & 84 & 81 & 3 \\
\hline 1 & 34 & 28 & 5 & 0 & -6 & 66 & 64 & 3 & 6 & 2 & 64 & 67 & 4 \\
\hline 2 & 80 & 86 & 3 & 0 & -4 & 110 & 113 & 4 & 7 & -5 & 128 & 128 & 4 \\
\hline 3 & 44 & 45 & 5 & 0 & -2 & 93 & 94 & 4 & 7 & -4 & 60 & 56 & 3 \\
\hline 4 & 60 & 61 & 4 & 0 & 0 & 157 & 164 & 4 & 7 & -3 & 49 & 49 & 4 \\
\hline-7 & 71 & 68 & 4 & 0 & 2 & 45 & 44 & 4 & 7 & -1 & 77 & 73 & 4 \\
\hline-5 & 74 & 73 & 3 & 1 & -7 & 93 & 87 & 4 & 7 & 1 & 90 & 90 & 4 \\
\hline-3 & 68 & 68 & 3 & 1 & -6 & 37 & 50 & 5 & 8 & -6 & 71 & 64 & 3 \\
\hline-1 & 75 & 75 & 3 & 1 & -5 & 59 & 60 & 3 & 8 & -5 & 43 & 37 & 4 \\
\hline 0 & 32 & 15 & 5 & 1 & -4 & 36 & 42 & 5 & 8 & -4 & 48 & 39 & 4 \\
\hline 1 & 164 & 171 & 4 & 1 & -3 & 255 & 254 & 5 & 8 & -2 & 61 & 55 & 4 \\
\hline 3 & 100 & 94 & 5 & 1 & -2 & 89 & 89 & 4 & 8 & 0 & 86 & 81 & 4 \\
\hline 4 & 65 & 66 & 4 & 1 & -1 & 87 & 79 & 4 & 8 & 1 & 73 & 71 & 3 \\
\hline-6 & 55 & 47 & 3 & 1 & 0 & 39 & 38 & 4 & 9 & -5 & 43 & 46 & 5 \\
\hline-5 & 37 & 39 & 5 & 1 & 1 & 111 & 119 & 4 & 9 & -3 & 114 & 110 & 4 \\
\hline-4 & 79 & 69 & 4 & 1 & 3 & 58 & 49 & 4 & 9 & -1 & 106 & 108 & 5 \\
\hline-3 & 55 & 56 & 3 & 2 & -6 & 125 & 118 & 4 & 9 & 0 & 73 & 63 & 4 \\
\hline-2 & 117 & 105 & 4 & 2 & -4 & 173 & 173 & 4 & 9 & 1 & 98 & 88 & 5 \\
\hline 0 & 152 & 148 & 4 & 2 & -3 & 104 & 108 & 4 & 10 & -4 & 118 & 119 & 4 \\
\hline 1 & 45 & 49 & 4 & 2 & -2 & 104 & 101 & 4 & 10 & -2 & 102 & 100 & 5 \\
\hline 2 & 85 & 81 & 3 & 2 & $-\overline{1}$ & 48 & 45 & 4 & 10 & 0 & 86 & 88 & 4 \\
\hline 3 & 40 & 36 & 5 & 2 & 0 & 158 & 163 & 4 & & & & & \\
\hline-7 & 70 & 61 & 4 & 2 & 2 & 60 & 64 & 3 & $\wedge \wedge \wedge$ & $\cdots \wedge$ & $h=$ & 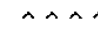 & $\wedge$ \\
\hline-5 & 131 & 130 & 4 & 2 & 3 & 56 & 56 & 4 & & & & & \\
\hline-3 & 231 & 228 & 5 & 3 & -7 & 129 & 128 & 4 & 0 & -6 & 48 & 46 & 4 \\
\hline-2 & 61 & 60 & 3 & 3 & -5 & 40 & 43 & 4 & 0 & -4 & 1.77 & 173 & 4 \\
\hline-1 & 117 & 119 & 4 & 3 & -4 & 72 & 67 & 3 & 0 & -2 & 100 & 92 & 4 \\
\hline 0 & 68 & 65 & 3 & 3 & -3 & 170 & 170 & 4 & 0 & 0 & 72 & 71 & 4 \\
\hline 1 & 134 & 128 & 4 & 3 & -2 & 46 & 46 & 4 & 0 & 2 & 73 & 78 & 4 \\
\hline 3 & 96 & 96 & 4 & 3 & -1 & 107 & 112 & 4 & 1 & -7 & 75 & 64 & 4 \\
\hline-6 & 79 & 82 & 4 & 3 & 1 & 86 & 89 & 4 & 1 & -5 & 67 & 68 & 3 \\
\hline-5 & 40 & 55 & 5 & 3 & 3 & 83 & 90 & 4 & 1 & -4 & 36 & 31 & 5 \\
\hline-4 & 113 & 114 & 4 & 4 & -7 & 60 & 68 & 4 & 1 & -3 & 113 & 103 & 4 \\
\hline-3 & 82 & 82 & 3 & 4 & -6 & 118 & 108 & 4 & 1 & -2 & 51 & 47 & 4 \\
\hline-2 & 133 & 132 & 4 & 4 & -5 & 33 & 26 & 5 & 1 & -1 & 123 & 122 & 4 \\
\hline 0 & 92 & 88 & 3 & 4 & -4 & 127 & 124 & 4 & 1 & 1 & 63 & 61 & 3 \\
\hline 2 & 122 & 114 & 4 & 4 & -3 & 39 & 37 & 5 & 1 & 2 & 45 & 36 & 4 \\
\hline-5 & 69 & 69 & 3 & 4 & -2 & 113 & 120 & 4 & 2 & -6 & 61 & 62 & 4 \\
\hline-3 & 59 & 56 & 3 & 4 & -1 & 44 & 47 & 4 & 2 & -4 & 74 & 72 & 4 \\
\hline-1 & 123 & 123 & 4 & 4 & 0 & 89 & 86 & 4 & 2 & -3 & 70 & 73 & 3 \\
\hline 0 & 41 & 35 & 5 & 4 & 2 & 76 & 78 & 4 & 2 & -2 & 56 & 57 & 4 \\
\hline 1 & 43 & 45 & 5 & 4 & 3 & 77 & 72 & 4 & 2 & 0 & 103 & 107 & 4 \\
\hline-4 & 110 & 111 & 4 & 5 & -7 & 57 & 55 & 4 & 2 & 2 & 53 & 50 & 4 \\
\hline-2 & 60 & 61 & 4 & 5 & -5 & 136 & 138 & 4 & 3 & -5 & 65 & 59 & 3 \\
\hline 0 & 48 & 47 & 4 & 5 & -2 & 84 & 88 & 4 & 3 & -4 & 83 & 83 & 4 \\
\hline-1 & 96 & 85 & 5 & 5 & -1 & 82 & 87 & 3 & 3 & -3 & 62 & 61 & 3 \\
\hline 0 & 55 & 49 & 4 & 5 & 0 & 80 & 82 & 3 & 3 & -1 & 83 & 84 & 4 \\
\hline
\end{tabular}




$$
\begin{aligned}
& 10|F| O \text { vs } 10 \mid \mathrm{F} / \mathrm{C} \text { for Quinolinic acid/Mn } \quad \text { page } 12 \\
& \text { k } 1 \text { FO FC sigF k } 1 \text { FO FC sigF k } 1 \text { Fo FC sigF } \\
& \begin{array}{rrrrrrrrrr}
3 & 0 & 35 & 30 & 5 & 4 & 0 & 55 & 55 & 4 \\
3 & 1 & 65 & 77 & 3 & 5 & -4 & 81 & 70 & 4 \\
3 & 2 & 40 & 19 & 4 & 5 & -3 & 105 & 103 & 5 \\
4 & -6 & 67 & 64 & 3 & 6 & -4 & 84 & 82 & 4 \\
4 & -4 & 64 & 58 & 3 & 6 & -2 & 93 & 79 & 5
\end{array}
\end{aligned}
$$

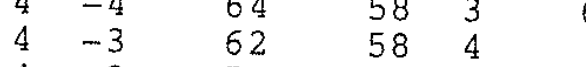

$$
\begin{aligned}
& \begin{array}{lllll}
4 & -2 & 51 & 45 & 4
\end{array} \\
& \begin{array}{rrrrr}
4 & 0 & 157 & 157 & 4 \\
5 & -5 & 97 & 96 & 5
\end{array}
\end{aligned}
$$

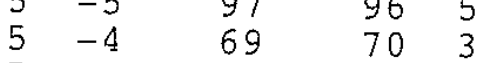

$$
\begin{aligned}
& \begin{array}{lllll}
5 & -3 & 102 & 94 & 5
\end{array} \\
& \begin{array}{lllll}
5 & -2 & 63 & 56 & 3
\end{array} \\
& \begin{array}{lllll}
5 & -1 & 66 & 69 & 3
\end{array} \\
& \begin{array}{lllll}
5 & 0 & 72 & 54 & 3
\end{array} \\
& \begin{array}{rrrrr}
5 & 1 & 76 & 81 & 4 \\
6 & -6 & 85 & 84 & 4
\end{array} \\
& \begin{array}{lllll}
6 & -6 & 85 & 84 & 4
\end{array} \\
& \begin{array}{lllll}
6 & -4 & 144 & 146 & 4
\end{array} \\
& \begin{array}{lllll}
6 & -2 & 108 & 107 & 5
\end{array} \\
& \begin{array}{rrrrr}
6 & 0 & 96 & 85 & 4 \\
7 & -5 & 98 & 94 & 5
\end{array} \\
& \begin{array}{rrrrr}
7 & -4 & 42 & 26 & 5
\end{array} \\
& \begin{array}{rrrrr}
7 & -3 & 140 & 136 & 5
\end{array} \\
& \begin{array}{rrrrr}
7 & -1 & 41 & 40 & 5 \\
8 & -4 & 50 & 43 & 4
\end{array} \\
& \begin{array}{lllll}
8 & -3 & 108 & 103 & 5
\end{array} \\
& \begin{array}{rrrrr}
8 & -2 & 126 & 122 & 5 \\
9 & -3 & 81 & 69 & 4
\end{array}
\end{aligned}
$$

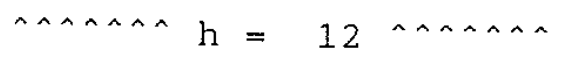

$$
\begin{aligned}
& \begin{array}{rrrrr}
0 & -6 & 65 & 63 & 4 \\
0 & -2 & 56 & 59 & 4 \\
0 & 0 & 112 & 107 & 4 \\
1 & -3 & 37 & 39 & 5 \\
1 & -1 & 156 & 150 & 4 \\
2 & -4 & 79 & 72 & 3 \\
2 & -3 & 51 & 47 & 4 \\
2 & -2 & 54 & 54 & 4 \\
2 & -1 & 53 & 49 & 4 \\
2 & 0 & 93 & 93 & 5 \\
3 & -5 & 120 & 113 & 5 \\
3 & -3 & 101 & 95 & 5 \\
3 & -2 & 37 & 28 & 5 \\
3 & -1 & 53 & 58 & 4 \\
3 & 0 & 39 & 34 & 5 \\
4 & -4 & 136 & 120 & 4 \\
4 & -3 & 38 & 38 & 6 \\
4 & -2 & 65 & 63 & 3 \\
4 & -1 & 47 & 47 & 4
\end{array}
\end{aligned}
$$

